\title{
IMAGE RESTORATION BY A FRACTIONAL REACTION-DIFFUSION PROCESS
}

\author{
HANA MATALLAH*, MESSOUAD MAOUNI, HAKIM LAKHAL \\ Laboratory of Applied Mathematics and History and Didactics of Mathematics "LAMAHIS", \\ Department of Mathematics, University 20 August 1955 Skikda, Algeria \\ *Corresponding author: hanamaatallah5@gmail.com
}

\begin{abstract}
We propose new approaches to the investigation of a reaction-diffusion model of fractional order in which we apply the fractional derivative in the sense of the Caputo by contribution to time on the model proposed by Nourddine Alaa in 2014, this study is based on the restoration of digital image such that a digital result is given on a noisy image in which this model is found to be effective in eliminating noise.
\end{abstract}

\section{INTRODUCTION}

Fractional order calculus is a topic which provides a good tool to describe physical memory and heredity, this topic has been applied to many fields such as flabby, oscillation, stochastic diffusion theory and wave propagation, biological materials, control and robotics, quantum mechanics, where it has also become used in the field of image processing. That field has become hot in recent years, and one of the topics recently included in this field is image processing or digital image processing, the latter is has become an important problem thanks to its wide importance in several fields and because this methode used to perform some operations on the digital image to get an enhanced image and to recovery lost information from it, and in this field we find a topic of The restoration of the image, which has become of interest to many researchers and scientists, where we find in 2019 [ [11], [12]] S. Lecheheb, M. Maouni and H. Lakhal, they proved the existence of the solution of a quasilinear equation and they give its application to image denoising and in the

Received April 22 ${ }^{\text {nd }}, 2021$; accepted May 26 ${ }^{\text {th }}, 2021$; published August $2^{\text {nd }}, 2021$.

2010 Mathematics Subject Classification. 35R11.

Key words and phrases. image restoration; reaction diffusion; fractional order derivative.

(C)2021 Authors retain the copyrights of their papers, and all open access articles are distributed under the terms of the Creative Commons Attribution License. 
same year, they used a nonlinear eliptic model in image restoration. In 2013 [14] M. Maouni and Nouri, used a new model based on p-gradient using to restore a digital image. Leheheb, Maouni and Lakhal, in 2021 [13], they used a novel model combining the Perona-Malik equation and the heat equation in image restoration, but the most problem in this topic that always arises in the form of question is how to treat the image while preserving edges but the answer of this quesion is given in 1987 [16] by Perona-Malik in his model, which is one of the first attempts to derive a model that incorporates local information from an image with in a partial differential equations $(P D E s)$, which the main reason for using $(P D E s)$ in this domain is that it is possible to analyse the image in continuous spaces. A nonlinear diffusion model, which they called's anisotropic diffusion was can ducted by Perona and Malik [16] in to reduce noise and enhance contrast while preserving the edge, but the basic Perona-Malik [16] PDE model is ill-posed in the sens of Hadamard. From where the idea of Catté, Lions, Morel and coll in 1992 [5] to integrate directly the regularisation into the equation by convolving the image with the gaussian filter on the gradient of the noisy image to smooth the image first in order to avoid the dependence of the numerical scheme between the solution and the regularization procedure to make the problem well posed and they prove existence, uniqueness and regularity for the related model and demonstrate experimentally that the related model gives similar results to the Perona-Malik equation [16]. In 2006 [15] Morfu proposed a model on the contrast enhancement and noise filtering where he combined the nonlinear diffusion process ruled by Fisher equation, his model is given as follows

$$
\begin{gathered}
\frac{\partial u}{\partial t}-\operatorname{div}(g(|\nabla u|) \nabla u)=f(u) \quad \text { in } Q_{T}, \\
u(0, x)=u_{0}(x) \quad \text { in } \Omega, \\
\frac{\partial u}{\partial v}=0 \quad \text { on } \Sigma_{T},
\end{gathered}
$$

with $\Omega$ is the domaine of the image, $\left.Q_{T}=\right] 0, T\left[\times \Omega, \Sigma_{T}=\right] 0, T\left[\times \partial \Omega\right.$, where $T>0, u_{0}$ is the original image and $f(s)=s(s-a)(1-s)$ with $0<a<1$.

The model of Morfu [15] has two disadvantage, the first one is the sensitivity to noise and the second is that no results of existence and consistency in proved. To overcome this problem, in 2014 [2] Alaa and all combining the regulaisation procedure in Catté [5] and Morfu [15] model, they proposed to modify the model of Morfu [15] by applying a gaussian filter on the gradient of the noisy image during the calculation of coefficient of anisotropic diffusion, the authors we reable to demonstrate the existence and consistency of the their proposed model, which is given by:

$$
\begin{gathered}
\frac{\partial u}{\partial t}-\operatorname{div}\left(g\left(\left|\nabla u_{\sigma}\right|\right) \nabla u\right)=f(t, x, u) \text { in } Q_{T}, \\
u(0, x)=u_{0}(x) \quad \text { in } \Omega, \\
\frac{\partial u}{\partial v}=0 \quad \text { on } \Sigma_{T}
\end{gathered}
$$


with $\Omega=] 0,1[\times] 0,1[$ the picture domain with boundary $\partial \Omega$, with Neumann boundary conditions, $u(t, x)$ is the restored image and $u_{0}$ is the original image to be processed. $\left.Q_{T}=\right] 0, T\left[\times \Omega, \Sigma_{T}=\right] 0, T[\times \partial \Omega,(T>0)$, $\sigma>0$ and $G_{\sigma}$ is the gaussian filter where:

$$
G_{\sigma}(x)=\frac{1}{\sqrt{2 \pi \sigma}} \exp \left(-\frac{|x|^{2}}{4 \sigma}\right), \quad x \in \mathbb{R}^{2} .
$$

They consider the gradient norm of $\omega$ as:

$$
|\nabla \omega|=\left(\sum_{i=1}^{i=2}\left(\frac{\partial \omega}{\partial x_{i}}\right)^{2}\right)^{1 / 2}
$$

$\nabla \omega_{\sigma}$ is the smoothed version of gradient norme where :

$\nabla \omega_{\sigma}=\nabla\left(\omega * G_{\sigma}\right)=\omega * \nabla G_{\sigma}$. The diffusivity $g$ is smooth decreasing function defined by

$$
g(0)=1, \lim _{s \longrightarrow \infty} g(s)=0,
$$

one of the diffusivities Perona and Malik [16] proposed is:

$$
g(s)=\frac{d}{\sqrt{1+\eta\left(\frac{s}{\lambda}\right)^{2}}},
$$

with $\eta \geq 0, d>0$ and $\lambda$ is a parameter that separates forward and backward diffusion [20]. In 2016, Bassam Al-Hamzah and Naji Yabari [4] proposed a new reaction-diffusion model in image processing, which they proved the existence of global solution for the nonlinear reaction-diffusion model. this study deal with the equation:

$$
\begin{gathered}
\frac{\partial u}{\partial t}-\operatorname{div}\left(g\left(\left|\nabla u_{\sigma}\right|\right) \nabla u\right)=f(t, x, u, \nabla u) \quad \text { in } Q_{T}, \\
u(0, x)=u_{0}(x) \geq 0 \quad \text { in } \Omega \\
\frac{\partial u}{\partial v}=0 \quad \text { on } \Sigma_{T}
\end{gathered}
$$

with $\Omega=] 0,1[\times] 0.1\left[, Q_{T}=\right] 0, T\left[\times \Omega\right.$ and $\left.\Sigma_{T}=\right] 0, T[\times \partial \Omega$.

The results $f(t, x, u, \nabla u)$ and $f=f(t, x, u)$ is a generalization of the work $f=0$ presented by Catté [5], and Alaa [2]. In 2018 Aaraba, Alaa, and Khalfi [1] provided the existence of global solution to a reaction-diffusion generic system with application in image restoration and anhancement. This study is a generalization of the work presented by [ [2], [5], [16]] in the case of reaction-diffusion equations. They give an example of application demonstrated on a novel bio-inspired image restoration model [1]. In the same year, Alaa and Zirhem [3], proposed a new model of nonlinear and anisotropic reaction diffudion system applied to image restoration and to contrast enhancement. This Model is based on a system of partial differential equations of type Fitzhugh-Nagumo. They compared the performance of their alghorithm with the classical FizhughNagumo model. 
The aim of this work is to show how fractional order differential equations are used to restore a digital image. It is afact to apply the fractional derivative in the sens of Caputo on the model the reaction-diffusion proposed with Alaa and all in 2014 [2], the proposed model is as follows:

$$
{ }_{0}^{C} \mathcal{D}_{t}^{\alpha} u(t, x)-\operatorname{div}\left(g\left(\left|\nabla u_{\sigma}\right|\right) \nabla u\right)=f(t, x, u) \quad \text { in } \quad Q_{T},
$$

with the conditions given by:

$$
\begin{gathered}
u(0, x)=u_{0} \quad \text { in } \quad \Omega \\
\frac{\partial u}{\partial \nu}=0 \quad \text { in } \quad \Sigma_{T},
\end{gathered}
$$

with $\left.0<\alpha<1, Q_{T}=\right] 0, T[\times \Omega$ and $\Omega=] 0,1[\times] 0,1[$ is the image domain with limit $\partial \Omega, u(t, x)$ is the solution to the problem, $u_{0}$ is the original image, $\nu$ is an outward normal to domain $\Omega$. Let $\sigma>0, \nabla u_{\sigma}$ be a regularization by convolution of $\left.\nabla u, \Sigma_{T}=\right] 0, T[\times \partial \Omega$, where $(T>0)$ and $0<t<T$, the diffusivity $g$ check the same properties provided by Alaa [2], which is given in the equation (1.3) and the function $f(t, x, u)$ is used to represent sources. ${ }_{0}^{C} \mathcal{D}_{t}^{\alpha} u(t, x)$ is the fractional derivative in the Caputo sense of order $\alpha$ of $u(t, x)$ defined as [10]:

$$
{ }_{0}^{C} \mathcal{D}_{t}^{\alpha} u(t, x)=\frac{1}{\Gamma(1-\alpha)} \int_{0}^{t} \frac{\partial}{\partial t} u(s, x)(t-s)^{-\alpha} d s \quad 0<\alpha<1 .
$$

In this study, we need the following assumptions and properties:

$\left(H_{1}\right)-f: Q_{T} \times \mathbb{R} \longrightarrow \mathbb{R}$ mesurable for $(t, x)$ and continous for $u$.

$\left(H_{2}\right)-\forall(t, x) \in Q_{T}, f(t, x, 0)$ is a positive function.

$\left(H_{3}\right)-\forall u \in \mathbb{R}$ and for all $(t, x) \in Q_{T}, u f(t, x, u)$ is negative.

$\left(H_{4}\right)$ - Assumed that $u(t, x)$ is differentiable in the sence of the gâteau(See [8], page 67), so

$$
{ }_{0}^{C} \mathcal{D}_{t}^{\alpha} u(t, x)=t^{1-\alpha} \frac{\partial u(t, x)}{\partial t}
$$

$\left(H_{5}\right)$ - Let $B(t)=t^{\alpha-1}$ and $\sup _{0<t<T}|B(t)| \leq C_{B}$, where $C_{B}>0$ and $0<\alpha<1$.

The equation(1.5) is given as follows:

$$
\begin{gathered}
\frac{\partial u}{\partial t}-B(t) \operatorname{div}\left(g\left(\left|\nabla u_{\sigma}\right|\right) \nabla u\right)=B(t) f(t, x, u) \quad \text { in } Q_{T}, \\
u(0, x)=u_{0}(x) \quad \text { in } \Omega \\
\frac{\partial u}{\partial v}=0 \quad \text { in } \Sigma_{T}
\end{gathered}
$$

In this case, we will recall some functional spaces that willbe used throughout this paper. $\forall k \in \mathbb{N}, H^{k}(\Omega)$ is the set of functions $u$ defined in $\Omega$ such as $u$ and its order $D^{m} u$ derivatives where $|m|=\sum_{n}^{j=1} m_{j} \leq k$ are 
in $L^{2}(\Omega) . H^{k}(\Omega)$ is Hilbert space with the norm

$$
\|u\|_{H^{k}(\Omega)}=\left(\sum_{|m| \leq k} \int_{\Omega}\left|D^{m} u\right|^{2} d x\right)^{\frac{1}{2}} .
$$

By setting $\left(H^{1}(\Omega)\right)^{\prime}$ the dual of $H^{1}(\Omega)$.

$L^{p}\left(0, T, H^{k}(\Omega)\right)$ is the space of functions $u$ such that, $\forall t \in(0, T), u(t)$ belongs to $H^{k}(\Omega)$ with the norm

$$
\|u\|_{L^{p}\left(0, T, H^{k}(\Omega)\right)}=\left(\int_{0}^{T}\|u(t)\|_{H^{k}(\Omega)}^{p} d t\right)^{\frac{1}{p}}, \quad 1<p<\infty, \quad k \in \mathbb{N} .
$$

$L^{\infty}\left(0, T, L^{2}(\Omega)\right)$ is the space of functions $u$ such that, $\forall t \in(0, T), u(t)$ belongs to $L^{2}(\Omega)$ with the norm

$$
\|u\|_{L^{\infty}\left(0, T, L^{2}(\Omega)\right)}=\left(\sup _{0<t<T}\|u(t)\|_{L^{2}(\Omega)}^{2}\right)^{\frac{1}{2}} .
$$

$L^{\infty}\left(0, T, C^{\infty}(\Omega)\right)$ is the space of funcions $u$ such that, for all every $t \in(0, T), u(t)$ belongs to $C^{\infty}(\Omega)$ with the norm

$$
\|u\|_{L^{\infty}\left(0, T, C^{\infty}(\Omega)\right)}=\inf \left\{c,\|u(t)\|_{C^{\infty}(\Omega)} \leq C \quad \text { in } \quad(0, T)\right\} .
$$

this study is based on the existence of the solution for our model, we truncate the equation and show that it can be solved in the sens of the Schauder fixed point theorem. Finally by making some estimations, we prove that the solution of the approximate problem converge to the solution of the aour our problem. We state this paper first by an introduction, then we give a definition of solution with the presentation of the most important results of this work, followed by a description of the existence of the reaction diffusion equation problem, finally we give a straightforward application of our result in the fractional reaction diffusion model for image restoration.

\section{THE MAIN RESUlT}

First, we clearly state our definition of weak solution to the reaction-diffusion equation, we define the folowing spaces:

$$
\begin{gathered}
X=\left\{u \text { in } L^{\infty}\left(0, T ; L^{2}(\Omega)\right) \cap L^{2}\left(0, T ; H^{1}(\Omega)\right), \quad u(0, .)=u_{0}\right\} . \\
Z=\left\{\varphi \text { in } C^{1}\left(Q_{T}\right), \text { where } \varphi(T, .)=0\right\} . \\
D=\left\{u \text { in } C\left([0, T] ; L^{2}(\Omega)\right) \cap L^{2}\left(0, T ; H^{1}(\Omega)\right\}\right.
\end{gathered}
$$

Definition 2.1. Let $u$ the weak solution of the problem (1.7) if

For all $u \in X$ and $\varphi \in Z$ with $f(t, x, u) \in L^{1}\left(Q_{T}\right)$

$$
\int_{Q_{T}}-u \frac{\partial \varphi}{\partial t} d x d t+\int_{Q_{T}} B(t) g\left(\left|\nabla u_{\sigma}\right|\right) \nabla u \nabla \varphi d x d t=\int_{Q_{T}} B(t) f(t, x, u) \varphi d x d t+\int_{\Omega} u_{0} \varphi(0, x) d x .
$$


Theorem 2.1. Under the assumption $\left(H_{1}\right)-\left(H_{5}\right)$ and for all $R \geq 0$,

$$
\sup _{|u| \leq R}(|f(t, x, u)|) \in L^{1}\left(Q_{T}\right) .
$$

Then with fixed $T>0, \sigma>0$ and $0<\alpha<1$ and for any $0 \leq u_{0} \in L^{2}(\Omega)$, the equation (2.1) admits a weak positive solution. If moreover for all $r \geq 1, f(t, x, r) \leq 0$ and $u_{0}(x) \leq 1$, we have $0 \leq u(t, x) \leq 1$ dans $Q_{T}$.

Proof of Theorem. The proof of Theorem(2.1) is done in four step:

Step1: The positivity of the solution. Let the function $\operatorname{sign}^{-}$defined as:

$$
\operatorname{sign}^{-}(r)=\left\{\begin{array}{lll}
-1 & \text { if } & r<0 \\
0 & \text { if } & r \geq 0 .
\end{array}\right.
$$

We build a sequence of convex function $j_{\varepsilon}(r)$ where $j_{\varepsilon}^{\prime}(r)$ is bounded and for all $r \in \mathbb{R}, j_{\varepsilon}^{\prime}(r)$ converge to $\operatorname{sign}^{-}(r)$ when $\varepsilon \longrightarrow 0$.

We consider $u$ the solution of (2.1), we multiply the equation by $j_{\varepsilon}^{\prime}(u)$ and by integration on $\left.Q_{t}=\right] 0, t[\times \Omega$ for $t \in[0, T[$ we get:

$$
\int_{Q_{t}} \frac{\partial u}{\partial t} j_{\varepsilon}^{\prime}(u) d x d s+\int_{Q_{t}} B(t) g\left(\left|\nabla u_{\sigma}\right|\right) \nabla u \nabla j_{\varepsilon}^{\prime}(u) d x d s=\int_{Q_{t}} B(t) f(s, x, u) j_{\varepsilon}^{\prime}(u) d x d s,
$$

we set $A(t, x)=g\left(\left|\nabla u_{\sigma}\right|\right)$ and with

$$
\left\|\nabla u_{\sigma}\right\|_{L^{\infty}\left(Q_{t}\right)} \leq C_{0}
$$

for the properties of $g$, we have $a=g\left(C_{0}\right)$ where $C_{0}$ depend to $\sigma$ and $\left\|u_{0}\right\|_{L^{\infty}}(\Omega)$, such that

$$
\begin{gathered}
A(t, x) \geq a \quad \forall(t, x) \in Q_{T} . \\
\int_{\Omega}\left[j_{\varepsilon}(u(t))-j_{\varepsilon}(u(0))\right] d x+a \int_{Q_{T}} B(t)|\nabla u|^{2} j_{\varepsilon}^{\prime \prime}(u) d x d t \leq \int_{Q_{T}} B(t) f(t, x, u) j_{\varepsilon}^{\prime}(u) d x d t,
\end{gathered}
$$

then

$$
\begin{aligned}
\int_{\Omega} j_{\varepsilon}(u(t)) d x & \leq C_{B} \int_{Q_{T}} f(t, x, u) j_{\varepsilon}^{\prime}(u) d x d t \\
& \leq C_{B} \int_{[u<0]} f(t, x, u) j_{\varepsilon}^{\prime}(u) d x d t+C_{B} \int_{[u \geq 0]} f(t, x, u) j_{\varepsilon}^{\prime}(u) d x d t
\end{aligned}
$$

then

$$
\int_{\Omega} j_{\varepsilon}(u(t)) \leq C_{B} \int_{[u<0]} f(t, x, u) j_{\varepsilon}^{\prime}(u) d x d t .
$$

By crossing in the limit, when $\varepsilon \rightarrow 0$

$$
\int_{\Omega}(u)^{-}(t) d x \leq-C_{B} \int_{[u \leq 0]} f(t, x, u) d x d t
$$


then $(u)^{-} \geq 0$, so $(u)^{-}=0$, hence $u \geq 0$.

Then, we have to prove the following lemma:

Lemma 2.1. We consider $u$ the weak solution of (2.1), and assume that $0 \leq u_{0} \leq 1$ in $\Omega$ then $0 \leq u \leq 1$ in $Q_{T}$.

Proof. In the previous results, we have obtained the positivity of the weak solution if the initial data is positive, so, we assume that $u_{0} \leq 1$ and prove that $u \leq 1$.

We take $\bar{u}=1-u$, where $\nabla \bar{u}=\nabla u$.

For all $\bar{u} \in X$ and $\varphi \in Z$ with $f(t, x, 1-\bar{u}) \in L^{1}\left(Q_{T}\right)$

$$
-\int_{Q_{T}} \bar{u} \frac{\partial \varphi}{\partial t} d x d t+\int_{Q_{T}} B(t) g\left(\left|\nabla \bar{u}_{\sigma}\right|\right) \nabla \bar{u} \nabla \varphi d x d t=\int_{Q_{T}} B(t) f(t, x, 1-\bar{u}) \varphi d x d t .
$$

Let $j_{\varepsilon}(r)$ a sequence of convex function, where $j_{\varepsilon}^{\prime}(r)$ is bounded and $\forall r \in \mathbb{R}, j_{\varepsilon}^{\prime}(r) \rightarrow \operatorname{Sing}^{-}(r)$ when $\varepsilon \rightarrow 0$, Let $j_{\varepsilon}^{\prime}(\bar{u})=\varphi$

$$
\begin{aligned}
& \int_{Q_{t}} \frac{\partial j_{\varepsilon}(\bar{u})}{\partial t} d x d s+\int_{Q_{t}} B(t) g\left(\left|\nabla \bar{u}_{\sigma}\right|\right) \nabla \bar{u} \nabla j_{\varepsilon}^{\prime}(\bar{u}) d x d s=\int_{Q_{t}} B(t) f(t, x, 1-\bar{u}) j_{\varepsilon}^{\prime}(\bar{u}) d x d s \\
& \int_{\Omega} j_{\varepsilon}(\bar{u}(t)) d x \leq C_{B} \int_{Q_{T}} f(t, x, 1-\bar{u}) j_{\varepsilon}^{\prime}(\bar{u}) d x d t \\
& \leq C_{B} \int_{[\bar{u}<0]} f(t, x, 1-\bar{u}) j_{\varepsilon}^{\prime}(\bar{u}) d x d t+C_{B} \int_{[\bar{u} \geq 1]} f(t, x, 1-\bar{u}) j_{\varepsilon}^{\prime}(\bar{u}) d x d t
\end{aligned}
$$

pass to the limit when $\varepsilon \rightarrow 0$

$$
-\int_{\Omega}(\bar{u})^{-}(t, x) \leq C_{B} \int_{[\bar{u} \geq 1]} f(t, x, 1-\bar{u}) j_{\varepsilon}^{\prime}(\bar{u}) d x d t
$$

Hence

$$
\int_{\Omega}(\bar{u})(t, x) d x \geq 0
$$

hence $(\bar{u}) \geq 0$, so $u=1-\bar{u} \leq 1$.

Step2: Existence result for bounded nonlinearity. First, we will show the existence result for bounded source term $f$.

Lemma 2.2. Under the above assumption of the nonlinearity $f$ and $\left(H_{5}\right)$, if there exists $M_{f} \geq 0$, for almost $(t, x) \in Q_{T}$ and every $r \in \mathbb{R}$, we have

$$
|f(t, x, r)| \leq M_{f}
$$

then for all $u_{0} \in L^{2}(\Omega)$, the problem (2.1) admits a weak solution. Moreover, there exists $C=C\left(M_{f}, a, T,\|u\|_{L^{2}(\Omega)}\right)$ where:

$$
\|u(t)\|_{L^{\infty}\left(0, T ; L^{2}(\Omega)\right)}+\|u\|_{L^{2}\left(0, T ; H^{1}(\Omega)\right)} \leq C .
$$


Proof. Firt, we introduce the space $W(0, T)$ to show the existence of a weak solution with the classical Schauder fixed point theorem:

$$
W(0, T)=\left\{v \in L^{2}\left(0, T ; H^{1}(\Omega)\right) \cap L^{\infty}\left(0, T ; L^{2}(\Omega)\right): \frac{\partial v}{\partial t} \in L^{2}\left(0, T ;\left(H^{1}(\Omega)\right)^{\prime}\right)\right\}
$$

Let $v \in W(0, T)$ and $u$ be the solution of a linearization of problem (1.7) given by

- $\forall u \in D$ and $\forall \varphi \in Z$

$$
\int_{Q_{T}}\left(-u \frac{\partial \varphi}{\partial t}+B(t) g\left(\left|\nabla v_{\sigma}\right|\right) \nabla u \nabla \varphi\right) d x d t=\int_{Q_{T}} B(t) f(t, x, v) \varphi d x d t+\int_{\Omega} u_{0} \varphi(0, x) d x .
$$

We take $u$ as a test function $\varphi$ in (2.10), with $0<t<T$

$$
\frac{1}{2} \int_{\Omega} u^{2}(t) d x+\int_{Q_{T}} B(t) g\left(\left|\nabla v_{\sigma}\right|\right)|\nabla u|^{2} d x d t=\int_{Q_{T}} B(t) f(t, x, v) u d x d t+\frac{1}{2} \int_{\Omega} u_{0}^{2} d x .
$$

with $(3.1)$ and $A(t, x)=g\left(\left|\nabla u_{\sigma}\right|\right) \geq a, \forall(t, x) \in Q_{T}$

$$
\int_{\Omega} u^{2}(t) d x+2 a \int_{Q_{T}}|B(t) \| \nabla u|^{2} d x d t \leq M_{f} \int_{Q_{T}} u^{2} d x d t+\int_{\Omega} u_{0}^{2} d x
$$

using Gronwall lemma

$$
\begin{gathered}
\int_{\Omega} u^{2}(t) d x \leq\left\|u_{0}\right\|_{L^{2}(\Omega)}^{2}\left(\exp \left(M_{f} T\right)-1\right) \\
\sup _{0<t<T} \int_{\Omega} u^{2}(t) d x \leq C_{1}
\end{gathered}
$$

then

$$
\|u\|_{L^{\infty}\left(0, T, L^{2}(\Omega)\right)} \leq C_{1} .
$$

From (2.11), we have

$$
2 a \int_{Q_{T}}|B(t)||\nabla u|^{2} d x d t+M_{f} \int_{Q_{T}}|u|^{2} d x d t \leq \int_{\Omega}|u(t)|^{2} d x+\int_{\Omega} u_{0}^{2} d x
$$

using $\left(H_{5}\right)$ we obtain that

$$
\begin{gathered}
\int_{Q_{T}}|\nabla u|^{2} d x d t+\int_{Q_{T}}|u|^{2} d x d t \leq \frac{\left\|u_{0}\right\|_{L^{2}(\Omega)}^{2}}{\min \left(2 a, M_{f}\right)}, \\
\|u\|_{L^{2}\left(0, T, H^{1}(\Omega)\right)} \leq C_{2}, \quad C_{2}>0 .
\end{gathered}
$$

From the previous estimates we introduce the space

$W_{0}(0, T)=\left\{v \in L^{2}\left(0, T, H^{1}(\Omega)\right) \cap L^{\infty}\left(0, T, L^{2}(\Omega)\right), v(0)=u_{0}\right.$ and $\left.\|u\|_{L^{2}\left(0, T, H^{1}(\Omega)\right)}+\|u\|_{L^{\infty}\left(0, T, L^{2}(\Omega)\right)} \leq C\right\}$, where $C\left(M_{f}, T, a,\left\|u_{0}\right\|_{L^{2}(\Omega)}\right)$. 
The space $W_{0}(0, T)$ is nonemply closed convex in $W(0, T)$, moreover it injects with a compact way in $L^{2}\left(0, T, L^{2}(\Omega)\right)$, we define the application

$$
\begin{array}{clc}
F: W_{0}(0, T) & \longrightarrow & W_{0}(0, T) \\
w & \longmapsto & F(w) .
\end{array}
$$

$F$ is well defined, to apply the Schauder fixed point theorem, we have to show that the application $F$ is weakly continuous from $W_{0}(0, T)$ in $W_{0}(0, T)$ we consider a sequence $v_{n} \in W_{0}(0, T)$ where $v_{n} \rightarrow v$ in $W_{0}(0, T)$ and let $u_{n}=f\left(v_{n}\right)$. According to the classical result of compactness, we can extract from the sequence $\left(u_{n}\right)$ a subsequence yet denoted $\left(u_{n}\right)$ such taht

- $u_{n} \rightarrow u$ weakly in $L^{2}\left(0, T ; L^{2}(\Omega)\right)$.

- $u_{n} \longrightarrow u$ strongly in $L^{2}\left(0, T ; L^{2}(\Omega)\right)$ and almost every where in $Q_{T}$.

- $\nabla u_{n} \rightarrow \nabla u$ weakly in $L^{2}\left(0, T ; L^{2}(\Omega)\right)$.

- $v_{n} \longrightarrow v$ strongly in $L^{2}\left(0, T ; L^{2}(\Omega)\right)$ and almost every where in $Q_{T}$.

- $\nabla G_{\sigma} * v_{n} \longrightarrow \nabla G_{\sigma} * v$ strongly in $L^{2}\left(0, T ; L^{2}(\Omega)\right)$ and almost every where in $Q_{T}$.

- $B(t) g\left(\left|\nabla G_{\sigma} * v_{n}\right|\right) \longrightarrow B(t) g\left(\left|\nabla G_{\sigma} * v\right|\right)$ strongly in $L^{2}\left(0, T ; L^{2}(\Omega)\right)$.

- $f\left(t, x, v_{n}\right) \rightarrow f(t, x, v)$ strongly in $L^{1}\left(Q_{T}\right)$.

- $B(t) f(t, x, v) \rightarrow B(t) f(t, x, v)$ strongly in $L^{1}\left(Q_{T}\right)$.

The latter is obtained by applying the dominated convergence theorem. We can then pass to the limit, then the sequene $u_{n}=F\left(v_{n}\right)$ converges weakly to $u=F(v)$ in $W_{0}(0, T)$, then we deduce the existence of $u \in W_{0}(0, T)$ such that $u=F(u)$ and thus the existence of $u \in W(0, T)$.

Step3: The truncated problem and a priori estimates. We consider the truncated function $\Psi_{n}$ in $C_{c}^{\infty}(\mathbb{R})$, such that $0 \leq \Psi_{n} \leq 1$ and defined by:

$$
\Psi_{n}(r)=\left\{\begin{array}{lll}
1 & \text { if } & |r| \leq n \\
0 & \text { if } & |r| \geq n+1
\end{array}\right.
$$

We truncate the nonlinearity $f$ by $\Psi_{n}$

$$
f_{n}\left(t, x, u_{n}\right)=\Psi_{n}(|u|) f(t, x, u)
$$

thus, we can earily check that $f_{n}$ satisfies $\left(H_{1}\right)-\left(H_{3}\right)$ with $M_{f}=M_{f n}$ and for every $(t, x) \in Q_{T}, \forall r \in \mathbb{R}$

$$
f_{n}\left(t, x, u_{n}\right) \rightarrow f(t, x, u)
$$

since $u_{0} \in L^{2}(\Omega)$ and $\left|f_{n}\left(t, x, u_{n}\right)\right| \leq M_{f n}$, so lemma (2.2) is applied, then we get the existence of a weak 
solution of the problem

$$
\begin{gathered}
\frac{\partial u_{n}}{\partial t}-B(t) \operatorname{div}\left(g\left(\mid \nabla\left(u_{n \sigma} \mid\right) \nabla u_{n}\right)=B(t) f_{n}\left(t, x, u_{n}\right) \quad \text { in } Q_{T}\right. \\
u_{n}(0, x)=u_{n_{0}} \quad \text { in } \Omega \\
\frac{\partial u_{n}}{\partial \nu}=0 \quad \text { in } \Sigma_{T}
\end{gathered}
$$

Now, we are in the case to prove that a subsequence $u_{n}$ converge to the weak solution $u$ of the problem (1.7), for this we have to prove that lemma:

Lemma 2.3. We consider $u_{n}$ as sequence of weak solutions given in (2.1):

(i) $\int_{Q_{T}}\left|f_{n}\left(t, x, u_{n}\right)\right| d x d t \leq \int_{\Omega}\left|u_{n 0}\right| d x$.

(ii) $u_{n}$ is bounded in $L^{2}\left(0, T, H^{1}(\Omega)\right)$ and

$$
\int_{Q_{T}}\left|u_{n} f_{n}\left(t, x, u_{n}\right)\right| d x d t \leq \int_{\Omega} u_{n 0}^{2} d x
$$

(iii) $u_{n}$ is relatively compact in $L^{2}\left(Q_{T}\right)$.

Proof. (i) We have:

$$
\begin{gathered}
\frac{\partial u_{n}}{\partial t}-B(t) \operatorname{div}\left(g\left(\mid \nabla\left(u_{n \sigma} \mid\right) \nabla u_{n}\right)=B(t) f_{n}\left(t, x, u_{n}\right)\right. \\
\int_{Q_{T}} \frac{\partial u_{n}}{\partial t} d x d t-\int_{Q_{T}} B(t) \operatorname{div}\left(g\left(\mid \nabla\left(u_{n \sigma} \mid\right) \nabla u_{n}\right) d x d t=\int_{Q_{T}} B(t) f_{n}\left(t, x, u_{n}\right) d x d t\right. \\
\int_{\Omega}\left|u_{n}(t)\right| d x+C_{B} \int_{Q_{T}}\left|f_{n}\left(t, x, u_{n}\right)\right| d x d t \leq \int_{\Omega}\left|u_{n 0}\right| d x \\
\int_{Q_{T}}\left|f_{n}\left(t, x, u_{n}\right)\right| d x d t \leq \int_{\Omega}\left|u_{n 0}\right| d x
\end{gathered}
$$

(ii) First, we prove that $u_{n}$ is bounded in $L^{2}\left(Q_{T}\right)$, for this, we consider $u_{n}$ as a test function $\varphi$ in the approximate problem

$$
\frac{1}{2} \int_{Q_{T}}\left|u_{n}\right|^{2}(t) d x+C_{B} a \int_{Q_{T}}\left|\nabla u_{n}\right|^{2} d x d t \leq C_{B} \int_{Q_{T}}\left|u_{n}\right|\left|f\left(t, x, u_{n}\right)\right| d x d t+\frac{1}{2} \int_{\Omega}\left|u_{n 0}\right|^{2} d x
$$

we have that $C_{B} a \int_{Q_{T}}\left|\nabla u_{n}\right|^{2} d x d t \geq 0$ then

$$
\int_{Q_{T}}\left|u_{n} f_{n}\left(t, x, u_{n}\right)\right| d x d t \leq \frac{1}{2} \int_{\Omega}\left|u_{n 0}\right| d x
$$

where

$$
\sup _{0<t<T}\left\|u_{n}(\Omega)\right\|_{L^{2}(\Omega)} \leq\left\|u_{n 0}\right\|_{L^{2}(\Omega)}^{2}
$$


Let from the previous result

$$
\begin{aligned}
& \frac{1}{2} \int_{\Omega} u_{n}^{2}(t) d x+a C_{B} \int_{Q_{T}}\left|\nabla u_{n}\right|^{2} d x d t \leq \frac{C_{B}}{2} \int_{Q_{T}} u_{n}^{2} d x d t+\left(\frac{C_{B}}{2}+\frac{1}{2}\right) \int_{\Omega} u_{n 0}^{2} \\
& \min \left(a C_{B}, \frac{C_{B}}{2}\right) \int_{Q_{T}}\left|\nabla u_{n}\right|^{2} d x d t+\int_{Q_{T}}\left|u_{n}\right|^{2} d x d t \leq\left(C_{B}+1\right) \int_{\Omega} u_{n 0}^{2} d x .
\end{aligned}
$$

Setting $C_{1}=\min \left(a C_{B}, \frac{C_{B}}{2}\right)$

$$
\begin{gathered}
\int_{Q_{T}}\left|\nabla u_{n}\right|^{2} d x d t+\int_{Q_{T}}\left|u_{n}\right|^{2} d x d t \leq C_{2} \int_{\Omega} u_{n 0}^{2} d x, \\
\left\|u_{n}\right\|_{L^{2}\left(0, T, H^{1}(\Omega)\right)} \leq C_{2}\left\|u_{n 0}\right\|_{L^{2}(\Omega)}^{2} .
\end{gathered}
$$

(iii) Let $f_{n}\left(t, x, u_{n}\right)$ bounded in $L^{1}\left(Q_{T}\right)$ and $\frac{\partial u_{n}}{\partial t}$ bounded in $L^{1}\left(0, T,\left(H^{1}(\Omega)\right)^{\prime}\right)$, with Simon [19], $u_{n}$ is relatively compact in $L^{2}\left(Q_{T}\right)$.

Step4: Convergence. According to the previous result of Lemma (2.3), $u_{n}$ is relatively compact in $L^{2}\left(Q_{T}\right)$, we can extract a subsequence still denoted $u_{n}$ where:

- $u_{n} \rightarrow u$ weakly in $L^{2}\left(0, T ; L^{2}(\Omega)\right)$ and almost every where in $Q_{T}$.

- $\nabla G_{\sigma} * u_{n} \longrightarrow \nabla G_{\sigma} * u$ strongly in $L^{2}\left(Q_{T}\right)$ and almost every where in $Q_{T}$.

- $B(t) g\left(\left|\nabla G_{\sigma} * u_{n}\right|\right) \longrightarrow B(t) g\left(\left|\nabla G_{\sigma} * u\right|\right)$ strongly in $L^{2}\left(Q_{T}\right)$.

- $f\left(t, x, v_{n}\right) \rightarrow f(t, x, v)$ for almost every where $Q_{T}$.

- $B(t) f(t, x, v) \rightarrow B(t) f(t, x, v)$ for almost every where in $Q_{T}$.

To prove that $u$ is a weak solution of (1.7), it suffices to prove that $f_{n}\left(t, x, u_{n}\right) \rightarrow f(t, x, u)$ in $L^{1}\left(Q_{T}\right)$, since $f_{n}\left(t, x, u_{n}\right) \rightarrow f(t, x, u)$ almost every where in $Q_{T}$. We will prove that $f_{n}\left(t, x, u_{n}\right)$ is uniformly integrable in $L^{1}\left(Q_{T}\right)$. For this we use the vitali theorem where:

$\forall \varepsilon>0, \exists \delta>0$, such that $\forall E \subset Q_{T}$ measurable with $|E|<\delta$ we have:

$$
\int_{E}\left|f_{n}\left(t, x, u_{n}\right)\right| \leq \varepsilon
$$

$\forall K \geq 0$ :

$$
\int_{E}\left|f_{n}\left(t, x, u_{n}\right)\right| d x d t \leq \int_{\left[E \cap\left|u_{n}\right| \leq K\right]}\left|f_{n}\left(t, x, u_{n}\right)\right| d x d t+\int_{\left[E \cap\left|u_{n}\right|>k\right]}\left|f_{n}\left(t, x, u_{n}\right)\right| d x d t .
$$

Where:

$$
\begin{gathered}
\int_{\left[E \cap\left|u_{n}\right| \leq K\right]}\left|f_{n}\left(t, x, u_{n}\right)\right| d x d t \leq \int_{E\left|u_{n}\right| \leq K} \sup _{n}\left|f_{n}\left(t, x, u_{n}\right)\right| d x d t \\
\int_{E}\left|f_{n}\left(t, x, u_{n}\right)\right| d x d t \leq \int_{E} \sup _{\left|u_{n}\right| \leq K}\left|f_{n}\left(t, x, u_{n}\right)\right| d x d t+\int_{\left[E \cap\left|u_{n}\right|>k\right]}\left|f_{n}\left(t, x, u_{n}\right)\right| d x d t
\end{gathered}
$$


we have that $\sup _{\left|u_{n}\right| \leq K}\left|f_{n}\left(t, x, u_{n}\right)\right| \in L^{1}\left(Q_{T}\right), \forall \varepsilon>0, \exists \delta>0$ such as $|E|<\delta$ then:

$$
\int_{E\left|u_{n}\right| \leq K} \sup _{1}\left|f_{n}(t, x, u)\right| d x d t \leq \frac{\varepsilon}{2}
$$

We have $\left|u_{n}\right|>K$

$$
\begin{aligned}
\int_{\left[E \cap\left|u_{n}\right|>K\right]}\left|f_{n}\left(t, x, u_{n}\right)\right| d x d t & \leq \frac{1}{K} \int_{Q_{T}} u_{n} f_{n}\left(t, x, u_{n}\right) d x d t \\
& \leq \int_{E\left|u_{n}\right| \leq K} \sup _{n}\left|f_{n}\left(t, x, u_{n}\right)\right| d x d t+\frac{1}{K} \int_{E \cap\left|u_{n}\right|>K}\left|u_{n} f_{n}\left(t, x, u_{n}\right)\right| d x d t \\
& \leq \frac{\varepsilon}{2}+\frac{1}{K} \int_{E}\left|u_{n} f_{n}\left(t, x, u_{n}\right)\right| d x d t
\end{aligned}
$$

if $K \geq \frac{\|u\|_{2}^{2}(\Omega)}{\varepsilon}$ then

$$
\int_{\left[E \cap\left|u_{n}\right|>K\right]}\left|f_{n}\left(t, x, u_{n}\right)\right| \leq \frac{\varepsilon}{2}
$$

hence

$$
\int_{E}\left|f_{n}\left(t, x, u_{n}\right)\right| \leq \varepsilon
$$

\section{The Application}

If $u(t, x)$ is differentiable in the sense of the gâteau, so ${ }_{0}^{C} \mathcal{D}_{t}^{\alpha} u(t, x)=t^{1-\alpha} \frac{\partial u}{\partial t}$, with $0<\alpha<1$. (See[ [8], page 67]).

So the problem (1.5) became:

$$
t^{1-\alpha} \frac{\partial u}{\partial t}-\operatorname{div}\left(g\left(\left|\nabla u_{\sigma}\right|\right) \nabla u\right)=\frac{2}{\Gamma(2.3)} \exp (x)(2-x) t^{1,3}-u(t, x)-2 \exp (x) t^{2},
$$

with $0<\alpha<1$. The explicit finite difference approximation for (3.1) is

$$
\begin{gathered}
t^{1-\alpha} \frac{u^{n+1}(i, j)-u^{n}(i, j)}{d t}-\operatorname{div}\left(g\left(\left|\nabla\left(G_{\sigma} * u^{n}(i, j)\right)\right|, \lambda^{n}\right) \nabla u^{n}(i, j)\right) \\
=\left(\frac{2}{\Gamma(2.3)} t^{-0.7}-1\right) u^{n}(i, j)-2 \exp (i, j) t^{2}
\end{gathered}
$$

with $d t$ is the time step, $0<t<T$ with $T$ is the processing time and $u_{i, j}^{n}$ is the approximation of $u(t, x)$ in the pixel $(i, j)$ in time $n d t$.

First of all, we consider an original image without noise then we apply the new model on a noisy image with an additive gaussian noise.

Figure 1. Cameraman image without noise. 


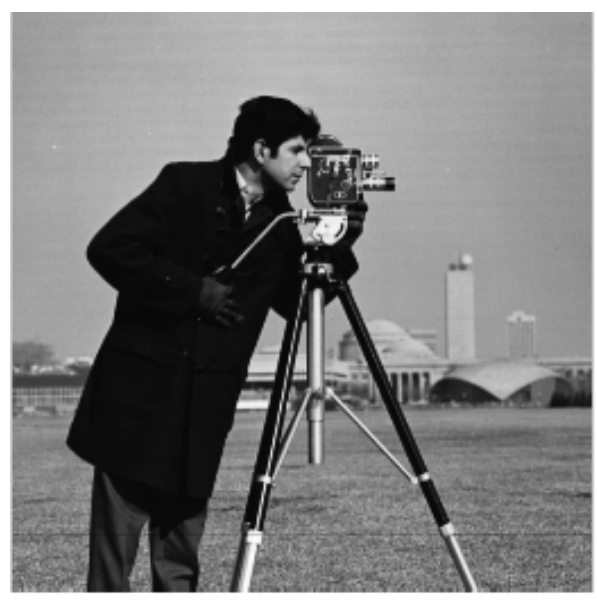

We apply an additive Gaussian noise on the binary image (1) with variance $\sigma=0.09$.

Figure 2. Noisy image with $\sigma=0.09$.

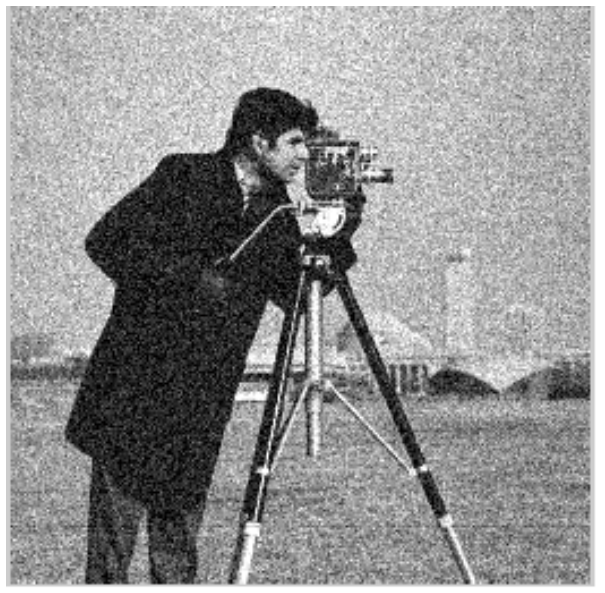

Then, we apply the fractional model on the noisy image (2), where we set the parameters of the model as follows:

we set the processing time $T$ at $0.009, t=0.001$ and $\alpha=0.7$.

Figure 3. Restored image with proposed model. 


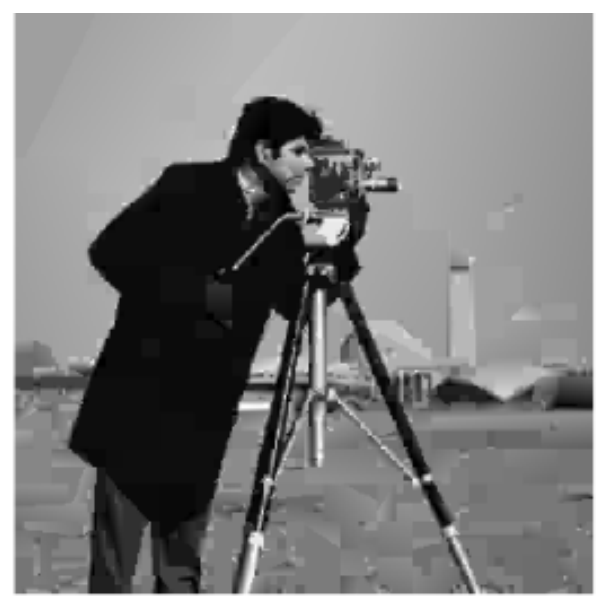

This fractional model can also be applied to the processing of color images.

Figure 4. Peppers image without noise.

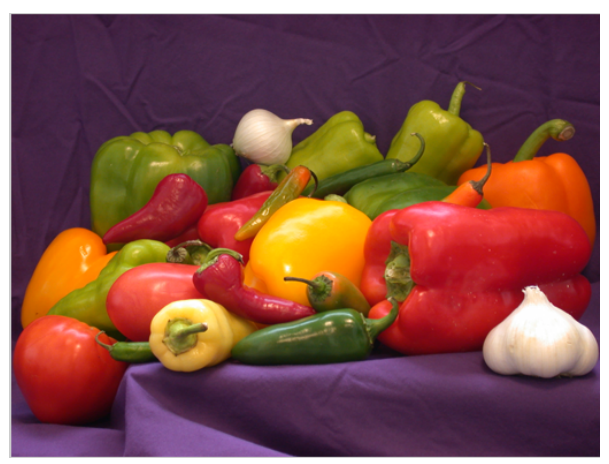

Figure 5. Noisy image with $\sigma=0.09$.

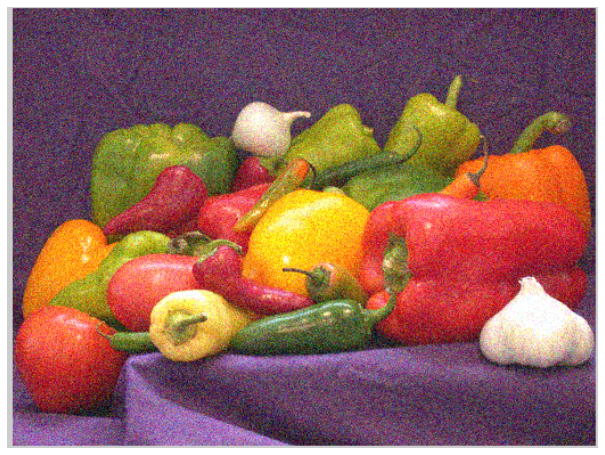

Figure 6. Restored image with proposed model. 


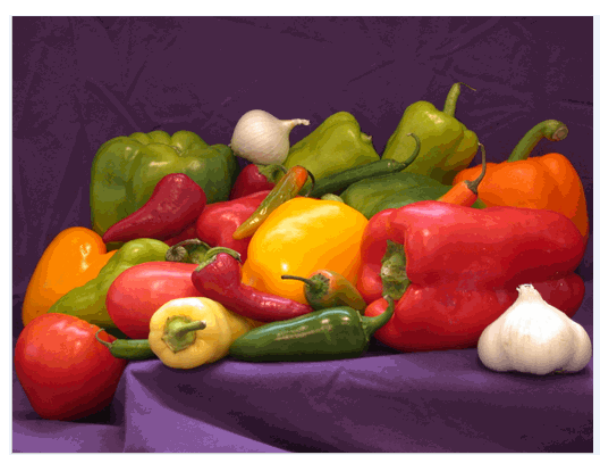

\section{The COnClusions}

In the case of summary, we demonstrated the existence of a global weak solution of the proposed model. Also, we proved that the truncated problem admits a weak solution according to Schauder fixed point theorem. For the nonlinear function satisfying suitable conditions, we established the equi-integrability and we derived a compactness result to be able to pass to the limit to get the desired result. To show the importance of the obtained result, a new application in the field of image restoration.

Conflicts of Interest: The author(s) declare that there are no conflicts of interest regarding the publication of this paper.

\section{REFERENCES}

[1] A. Aarab, N. Alaa, H. Khalfi. Generic reaction-diffusion model with application to image restoration and enhancement, Electron. J. Differ. Equ. 2018 (2018), 125.

[2] N. Alaa, M. Aitoussous, W. Bouarifi, D. Bensikaddour, Image restoration using a reaction-diffussion process, Electron. J. Differ. Equ. 2014 (2014), 197.

[3] N.E. Alaa, M. Zirhem, Bio-inspired reaction diffusion system applied to image restoration, Int. J. Bio-Inspired Comput. 12 (2018), 128-137.

[4] B. Al-Hamzah, N. Yebri. Global existence in reaction diffusion nonlinear parabolic partial differential equation in image procrssing, Glob. J. Adv. Eng. Technol. Sci. 3 (2016), 5.

[5] F. Catté, P.L. Lions, J-M. Morel, T. Coll. Image selective smoothing and edge detection by nonlinear diffusion. SIAM J. Numer. Anal. 29 (1992), 182-193.

[6] J. Chen. An implicit approximation for the Caputo fractional reaction-dispersion equation. J. Xiamen Univ. (Nat. Sci.) 46 (2007), 616-619. (in Chinese).

[7] C. Gong, W. Bao, G. Tang, Y. Jiang, J. Liu, A domain decomposition method for time fractional reaction-diffusion equation, Sci. World J. 2014 (2014), 681707.

[8] R. Khalil, M. Al Horani, A. Yousef, M. Sababheh, A new definition of fractional derivative, J. Comput. Appl. Math. 264 (2014), 65-70.

[9] A. Kilbas, H.M. Srivastava and J.J. Trujillo. Theory and Applications of Fractional Differential Equations. volume 204 of North-Holland Mathematics Studies. Elsevier, Amesterdam, 2006. 
[10] R. Khalil, M. Al Horani, A. Yousef, M. Sababheh, A new definition of fractional derivative, J. Comput. Appl. Math. 264 (2014), 65-70.

[11] S. Lecheheb, M. Maouni, H. Lakhal, Existence of the solution of a quasilinear equation and its application to image denoising, Int. J. Comput. Sci. Commun. Inform. Technol. 7 (2019), 1-6.

[12] S. Lecheheb, M. Maouni, H. Lakhal, Image restoration using nonlinear elliptic equation, Int. J. Comput. Sci. Commun. Inform. Technol. 6 (2019), 32-37.

[13] S. Lecheheb, M. Maouni, H. Lakhal, Image restoration using a novel model combining the Perona-Malik equation and the heat equation, Int. J. Anal. Appl. 19 (2021), 228-238.

[14] M. Maouni, F.Z. Nouri, Image restoration based on p-gradient model, Int. J. Appl. Math. Stat. 41 (2013), 48-57.

[15] S. Morfu. On some applications of diffusion processes for image processing. Phys. Lett. A, 373 (2009), 24-44.

[16] P. Perona, J. Malik, Scale-space and edge detection using anisotropic diffusion, IEEE Trans. Pattern Anal. Mach. Intell. $12(1990), 629-639$.

[17] B. Tellab, Résolution des équations différentielles fractionnaires. Université des Frères Mentouri Constantine-1, 2018.

[18] Feng Xing. Méthode de décomposition de domaines pour l'équation de Schrödinger. Analyse numérique [math.NA]. Université Lille 1 - Sciences et Technologies, 2014.

[19] J. Simon, Compact sets in the spaceL p (O,T; B), Ann. Mat. Pura Appl. 146 (1986), 65-96.

[20] J. Weickert. Anisotropic Diffusion in Image Processing. PhD Thesis, Kaiserslautern University, Kaiserslautern, Germany, 1996.

[21] F.Z. Zeghbib, M. Maouni, F.Z. Nouri, Overlapping and nonoverlapping domain decomposition methods for image restoration, Int. J. Appl. Math. Stat. 40 (2013), 123-128. 